Conclusions The combination of PF-8600 and uto had a tolerable safety profile and demonstrated clinical benefit, including in an NSCLC patient who had progressed on anti-PD1 therapy and achieved a durable partial response. Further combinations with one or both of these immune costimulatory receptor agonist antibodies might enhance their efficacy.

Acknowledgements This study was funded by Pfizer Inc. Editorial support was provided by Chu Kong Liew, PhD, of Engage Scientific Solutions and was funded by Pfizer Inc.

Trial Registration ClinicalTrials. gov: NCT02315066

Ethics Approval The study was approved by the institutional review board at each study center and conducted in accordance with the ethical principles of the Declaration of Helsinki.

\section{P862 CLINICAL BENEFIT POTENTIALLY EVIDENT WITH IMMUNOPHARMACODYNAMIC RESPONSES IN PRIOR- CHECKPOINT FAILED METASTATIC MELANOMA PATIENTS TREATED WITH IMPRIME PGG AND PEMBROLIZUMAB}

\begin{abstract}
${ }^{1}$ Anissa Chan, ${ }^{1}$ Nandita Bose*, ${ }^{1}$ Nandita Bose, ${ }^{1}$ Nadine Ottoson, ${ }^{1}$ Xiaohong Qiu, ${ }^{1}$ Ben Harrison, ${ }^{1}$ Richard Walsh, ${ }^{1}$ Paulette Mattson, ${ }^{1}$ Michele Gargano, 'Joanna Cox, ${ }^{2}$ Michael Chisamore, ${ }^{1}$ Mark Uhlik, 'Jeremy Graff. 'Biothera Pharmaceuticals, Eagan, MN, USA; ${ }^{2}$ Merch and Co., Inc., Rahway, NJ, USA
\end{abstract}

10.1136/LBA2019.15

Background Checkpoint inhibitor (CPI) monotherapy has revolutionized the treatment of melanoma, yet most patients are primary nonresponders or develop secondary resistance. Lack of antigen-specific $\mathrm{T}$ cell priming and/or immunosuppressive mechanisms leading to $\mathrm{T}$ cell exhaustion are critical cancerextrinsic factors contributing to CPI resistance mechanisms. Immunotherapeutic agents capable of sparking de novo antitumor $\mathrm{T}$ cell responses or reinvigorating pre-existing exhausted $\mathrm{T}$ cell immunity could help reinstate the activity of CPI.

Methods Our Phase 2, multi-center, open label study, NCT02981303 in collaboration with Merck \& Co., Inc., is evaluating Imprime PGG (Imprime), a novel yeast derived, Dectin-1 agonist, $\beta$-glucan PAMP in combination with pembrolizumab (KEYTRUDA ${ }^{\circledR}$, pembro) in heavily CPI pre-treated melanoma patients (20 patients; $65 \%$ had $>2$ prior CPI regimens with $17 / 20$ having previously progressed on pembro). Patients received Imprime $(4 \mathrm{mg} / \mathrm{kg})+$ pembro $(200 \mathrm{mg})$ intravenously in a 3-week cycle. Here, we present the immunopharmacodynamic (IPD) responses elicited by Imprime and pembro in the peripheral blood of 19 patients.

Results In the intent-to-treat population (ITT; $\mathrm{N}=20$ ), the disease control rate was 45\% (1 CR and 8 SD), 6-month and 12 -month OS rates were $65 \%$ and $45 \%$ respectively, and median OS (mOS) was 8.8 months. In the patients showing disease control, a significant increase in $\mathrm{CH} 50$, the classical pathway complement function ( 0.7-2.6-fold), HLA-DR expression on classical monocytes ( $\sim 0.61-1.94$-fold $)$ and reduction of frequency of PD-1+Tbet-EOMES + exhausted CD8 $T$ cells $(\sim 0.9-4$-fold $)$ was observed. Stimulation of peripheral blood mononuclear cells from a subset of patients by $\mathrm{CD} 3 / \mathrm{CD} 28$ beads showed enhanced production of IL-2 and IFN-gamma in the CD8 T cells. Some of these IPD responses were also associated with 6-month landmark OS analyses. Additionally, whole blood gene expression analyses showed $>2$-fold upregulation of several myeloid and $\mathrm{T}$ cell activation genes including IFNg, CD83, IP-10, and IL-2RA. Enhanced OS was observed in patients with $>1.3$ fold increase in CH50 (8/19; HR $0.385 ; \mathrm{p}=0.1)$ or $>1.5$-fold reduction in the frequency of exhausted CD8 T cells $(8 / 19$; HR 0.102; $p=0.001)$. The IPD responses observed in the ITT population included formation of circulating immune complexes (peak levels ranging from $\sim 4.5-16.1$-fold) and production of complement activation protein SC5b9 ( 3.4-25.6-fold), and increase in the frequency of HLA-DR+ myeloid cells ( 0.43-3.71-fold).

Conclusions Overall, these data, albeit in a small population, demonstrate that Imprime/pembro combination can drive the innate/adaptive IPD responses that are critical for providing clinical benefit to the patients who have progressed through prior CPI treatments.

Ethics Approval The study was approved by central and local ethics committees depending on site requirements. The central IRB for the study is Western Institutional Review Board (WIRB), approval number 20162506; all sites received IRB approval before opening the study at the respective sites.

\section{P863 KEYNOTE-022 PARTS 4 AND 5: PEMBROLIZUMAB PLUS TRAMETINIB FOR PATIENTS WITH SOLID TUMORS OR BRAF WILD-TYPE MELANOMA}

${ }^{1}$ Michele Maio*, ${ }^{2}$ Matteo Carlino, ${ }^{3}$ Anthony Joshua, ${ }^{4}$ Elaine McWhirter, ${ }^{5}$ Antoni Ribas, ${ }^{6}$ Paolo Ascierto, ${ }^{7}$ Wilson Miller, ${ }^{3}$ Marcus Butler, ${ }^{8}$ Pier Ferrucci, ${ }^{9}$ Robert Zielinski, ${ }^{10}$ Michele Del Vecchio, ${ }^{11}$ Eduard Gasal, ${ }^{12}$ Razi Ghori, ${ }^{12}$ Scott Diede, ${ }^{12}$ Elizabeth Croydon, ${ }^{13}$ Omid Hamid. 'Center for Immuno-Oncology, University Hospital of Siena, Siena, Italy; ${ }^{2}$ Melanoma Institute Australia, The University of Sydney, Westmead and Blacktown Hospitals, Sydney, NSW, Australia; ${ }^{3}$ Kinghorn Cancer Centre, St. Vincent's Hospital, Sydney, NSW, Australia; ${ }^{4}$ Juravinski Cancer Centre, McMaster University, Hamilton, ON, Canada; ${ }^{5}$ David Geffen School of Medicine, University of California, Los Angeles, Los Angeles, CA, USA; ${ }^{6}$ Istituto Nazionale Tumori IRCCS 'Fondazione G. Pascale', Naples, Italy; 'Lady Davis Institute for Medical Research, Jewish General Hospital, and McGill University, Montreal, QC, Canad; ${ }^{8}$ Istituto Europeo di Oncologia - IRCCS, Milan, Italy; ${ }^{9}$ Buffalo Medical Group, Williamsville, NY, USA; ${ }^{10}$ Fondazione IRCCS Istituto Nazionale, Milan, Italy; ${ }^{11}$ Novartis, East Hanover, NJ, USA; ${ }^{12}$ Merck and Co., Inc., Kenilworth, NJ, USA; ${ }^{13}$ The Angeles Clinic and Research Institute, Los Angeles, CA, USA

\subsection{6/LBA2019.16}

Background Pembrolizumab + dabrafenib + trametinib demonstrated promising antitumor activity and acceptable tolerability in BRAF-mutant melanoma in phase $1 / 2$ KEYNOTE-022 parts 1 and 2 (NCT02130466). Pembrolizumab+dabrafenib + trametiinib numerically prolonged PFS and DOR versus placebo+dabbrafenib + trametinib but had a higher grade 3-5 TRAE rate in part 3. KEYNOTE-022 parts 4 and 5 evaluated pembrolizumab+trametinib.

Methods In part 4 (open-label, $3+3$ dose-finding) patients with advanced solid tumors (irrespective of BRAF status) or unresectable/metastatic BRAF wild-type melanoma received pembrolizumab $200 \mathrm{mg}$ Q3W with trametinib as concurrent ( 2 or 4 weeks of trametinib run-in [1.5 or $2 \mathrm{mg} \mathrm{QD}]$, then pembrolizumab+trametinib [1.5 or $2 \mathrm{mg} \mathrm{QD}]$ ) or intermittent dosing ( 2 weeks of trametinib run-in [1.5 or $2 \mathrm{mg} \mathrm{QD}]$, then pembrolizumab+trametinib [1.5 or $2 \mathrm{mg}$ QD; 1 week off/2 weeks on]). Interim MTDs identified in part 4 were confirmed in part 5 using a modified toxicity probability interval design. The primary objectives were safety, tolerability, and ORR by investigator assessment per RECIST v1.1 of the maximum administered or tolerated dose (MAD/MTD) of 